\title{
Surface replication with one-nanometer-level smoothness by a nickel electroforming process
}

\author{
Hidekazu Mimura****, Satoshi Matsuyama***, Yasuhisa Sano***, Kazuto Yamauchi*** \\ (Received August 30, 2010)
}

\author{
*The University of Tokyo, 7-3-1 Hongo, Bunkyo-ku, Tokyo 113-8656, Japan \\ ** PRESTO, Japan Science and Technology Agency, 4-1-8 Honcho, Kawaguchi-shi, \\ Saitama 332-0012, Japan \\ *** Osaka University, 2-1 Yamada-oka, Suita, Osaka 565-0871, Japan
}

\begin{abstract}
A surface replication process is necessary for the mass production of nanometer-sized patterned and/or nanometer-flat surfaces. We have been developing a surface replication technique, aiming for 1 nanometer level replication accuracy in height direction. The process is based on nickel electroforming under low-temperature conditions. To investigate replication accuracy, we used an EEM (elastic emission machining) processed surface, having $0.1 \mathrm{~nm}$ flatness in root-mean-square (RMS) as a master surface, and compared the roughness of the master and replicated surfaces. The surface roughness is evaluated by using phase-shift microscopic interferometry and atomic force microscopy. The results indicate that surface replication is possible for one-nanometer-level smoothness. The durability of the master surface and repeatability of the process are also investigated. Surface quality of the master surface was found to be maintained during multiple electroforming processes.
\end{abstract}

Key words: Electroforming, surface smoothness, optical surface, elastic emission machining

\section{INTRODUCTION}

Electroforming is based on electrodeposition, which has been an important process in industry for a long time, and is used for the mass production of products with complicated shapes. Recently, electroforming has begun to be used in microelectromechanical systems (MEMS) for replicating surfaces with micrometer or nanometer-size patterns ${ }^{1)-3}$. Nickel is widely employed as a material in electroforming because high-quality $\mathrm{Ni}$ films can be easily obtained and corrosion can be prevented on materials by using $\mathrm{Ni}$ film coatings ${ }^{4)-5)}$.

In the conventional electroforming process, the separation method is key for realizing a highly accurate process; the electrodeposited product can be separated from the master surface only after electrodeposition, not during electrodeposition. After electrodeposition is finished, it is important that the replicated surface can be separated smoothly intentionally. In almost every current electroforming method, prior to electrodeposition, an organic interlayer film a few nanometers thick is formed by a wet process to cover the master surface. This is to prevent contact between the electrodeposited film and the master surface. When the replicated product is separated from the master surface, part of the interlayer film is destroyed. However, in this process, since the replicated surface is not in direct contact with the master surface, the surface morphology is not the same as that of the master surface.
Recently, we proposed and developed an electroforming process for the replication of surfaces having nanometer-level smoothness ${ }^{6)}$. A crucial aspect of this process is the method of depositing metal films as an electrode on a master surface. In this process, chromium was deposited by arc plasma deposition to act as a binding material. Subsequently, a nickel film was deposited by electron beam deposition to form the electrode. Electrodeposition was then carried out in a nickel sulfamate bath. By controlling the density of $\mathrm{Cr}$ atoms on the master surface, the binding power between the nickel film and master surface can be adjusted, which makes it possible to separate the metal film smoothly from the master surface.

For the progress of this replication process, it is necessary to analyze differences between the replicated and master surfaces. When a relatively rough surface is used as a master, the replication performance in terms of smoothness cannot be determined clearly because slight differences between the two surfaces is not distinguishable.

In this study, an atomic-level flat surface was prepared by elastic emission machining (EEM) and used as the master surface. The surface roughnesses of the master and replicated surfaces were compared and analyzed using power spectral density profiles ${ }^{7)}$. Multiple electroforming processes were also carried out to investigate the durability of the master surface as well as the repeatability. In this paper, the performance of the replication process is discussed on the basis of the observed results. 


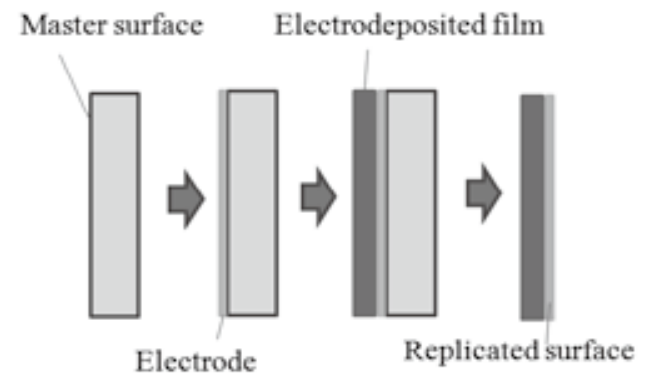

Fig. 1 Procedure of the electroforming process. The process includes electrode preparation, electrochemical deposition, and a separation process.

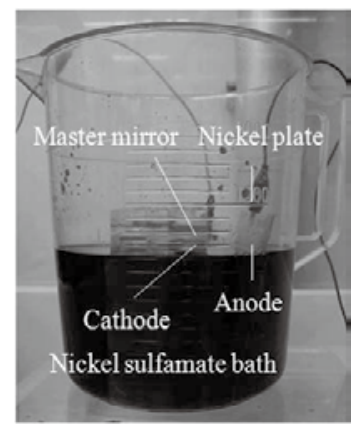

Fig. 2 Photograph of the electrochemical deposition process. A nickel sulfamate bath is employed. Direct current voltage with ON/OFF pulse is supplied between the anode and cathode.

Table1 Experimental conditions of electrodeposition

\begin{tabular}{cc}
\hline Bath solution & nickel sulfamate \\
& $(1.5 \mathrm{~mol} / \mathrm{l})$ \\
Temperature & 25 degree \\
Electrode material & Nickel \\
Electric voltage & $1.8 \mathrm{~V}$ \\
Power current density & $1 \mathrm{~mA} / \mathrm{cm}^{2}$ \\
Pulse interval & On $: 2 \mathrm{~s}, \mathrm{Off}: 3 \mathrm{~s}$ \\
Area & $5 \times 70 \mathrm{~mm}^{2}$ \\
\hline
\end{tabular}

\section{ELECTROFORMING PROCESS}

The proposed process includes electrode layer deposition, electrodeposition, and separation, in sequence as shown in fig. 1. The point of this process is the use of a $\mathrm{Cr}$ layer as a binder between master and metal in forming an electrode layer. First, $\mathrm{Cr}$ atoms were deposited by a vacuum arc plasma method. Cr chemically bonds to both the $\mathrm{SiO}_{2}$ surface used for the master surface and the metal film, which means that $\mathrm{Cr}$ acts as a binder material between $\mathrm{SiO}_{2}$ and the metal. Second, a nickel film with a thickness of approximately 100 $\mathrm{nm}$ is deposited by electron beam deposition evaporation. This film is used as the electrode in the subsequent metallic electrodeposition process. Then, nickel with a thickness of approximately $100 \mu \mathrm{m}$ is electrodeposited by applying a voltage in a nickel sulfamate bath, as shown in fig. 2. Table 1 shows typical experimental conditions. Finally, the nickel film including the electrode layer is mechanically separated from the master surface.

The density of $\mathrm{Cr}$ atoms on the master surface is adjusted so as not to separate the Ni film during the electrodeposition process, but to be able to separate the Ni film after the electrodeposition. The density of the $\mathrm{Cr}$ used was only $100 \times 10^{12}$ atoms $/ \mathrm{cm}^{26)}$. It is to be noted that $\mathrm{Cr}$ atoms did not cover the entire area of the master surface, and $90 \%$ of the replicated surface area was in direct contact with the master surface. Consequently, the same surface smoothness of the replicated surface as that of the master surface could be natively obtained.

\section{SURFACE ROUGHNESS EVALUATION}

EEM is one of the surface smoothing methods which can produce atomically flat surfaces. EEM is currently widely used for the production of advanced mirror devices installed in synchrotron radiation facilities and in extreme ultraviolet lithography ${ }^{7)}$. This flatness obtained using EEM is thus effective in the analysis of replication performance.

In the present work, a commercially obtained polished quartz surface was smoothed by EEM. Figure 3(a) shows the height map and fig. 3(b) shows the cross-sectional profile of line A-A' in fig. 3(a). The shown area is $64 \mu \mathrm{m} \times 48 \mu \mathrm{m}$. This map was obtained by a phase-shift microscopic interferometer (ZYGO, NewView200CHR). The root-mean-square (RMS) value is $0.09 \mathrm{~nm}$. The cross-sectional profile in fig. 3(b) also indicates the $0.1-n m-l e v e l$ flatness. The EEM processed surface was replicated, following the procedures in fig. 1 . Figure 4 shows the surface height map of the replicated surface. The RMS value of this map is $0.22 \mathrm{~nm}$.

In order to investigate the microroughness, the master and replicated surfaces were evaluated by atomic force microscopy (AFM: SII NanotechnologySPA-400). Figure 5 shows the three-dimensional height maps. RMS values of the master and replicated surfaces are 0.10 and $0.18 \mathrm{~nm}$, respectively. These results indicate that the quality of surface roughness of the replicated surface was lower than that of the master surface, however, highly accurate replication at the one-nanometer level is possible. 


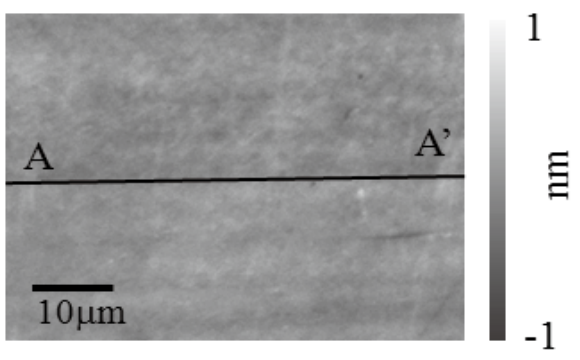

(a) Height map

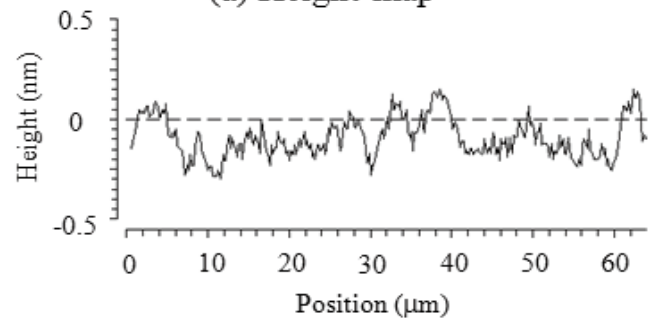

(b) Cross sectional profile

(A-A' in (a))

Fig. 3 Height profile maps of the master surface. These are obtained by phase-shift microscopic interferometry.

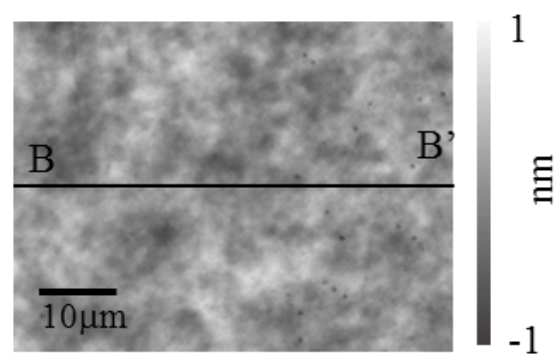

(a) Height map

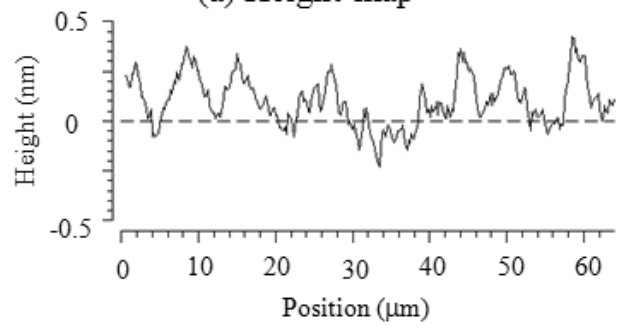

(b) Cross sectional profile

(B-B' in (a))

Fig. 4 Height map and cross sectional profile of a replicated surface. These are obtained by phase-shift microscopic interferometry.

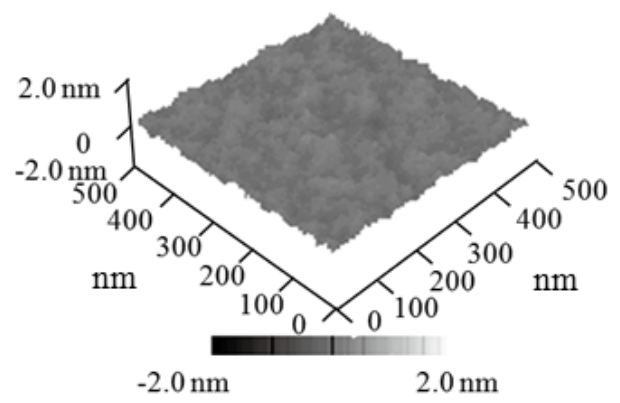

(a) Master surface

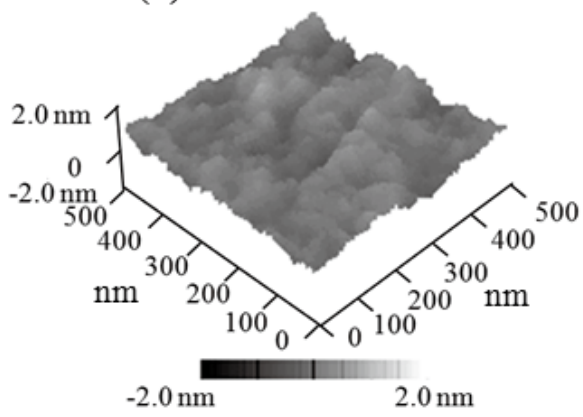

(b) Replicated surface

Fig. 5 Surface height profile maps of (a) the master and (b) the replicated surfaces. These profiles were obtained by atomic force microscopy.

\section{REPEATABILITY}

For multiple reproductions, the master surface needs to be used a number of times. In this study, a second surface replication was carried out following the procedure in fig. 6 using the same master surface. After the first replication process, duties such as particles and dried bath solution are attached to the master surface. The master surface was treated with a wet cleaning process, called SPM cleaning. The second replication was then carried out under the same conditions as the first. Figure 7 shows surface height maps of the master surface after the first replication and the replicated surface obtained by the second replication. The RMS values of the master and replicated surface are 0.08 and $0.24 \mathrm{~nm}$, respectively. Thus, the surface flatness of the master surface was maintained after the first replication process, and the same quality replicated surface as in the first replication was obtained.

\section{CONCLUSION AND DISSCUSSION}

In this study, since an atomically flat surface was employed as a master surface, a slight difference in the surface roughness between the master and the replicated surfaces is clear. Thus, a flat surface with 


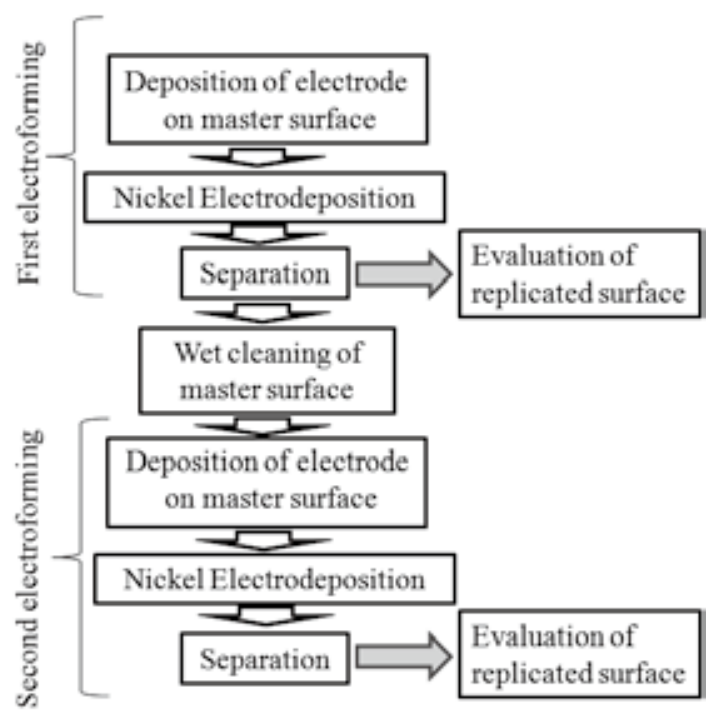

Fig. 6 Procedure for evaluation of repeatability

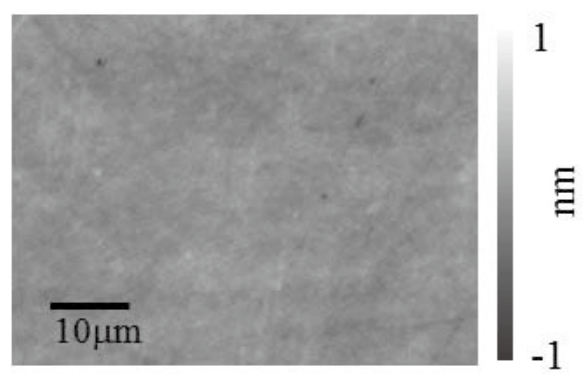

(a) Master surface

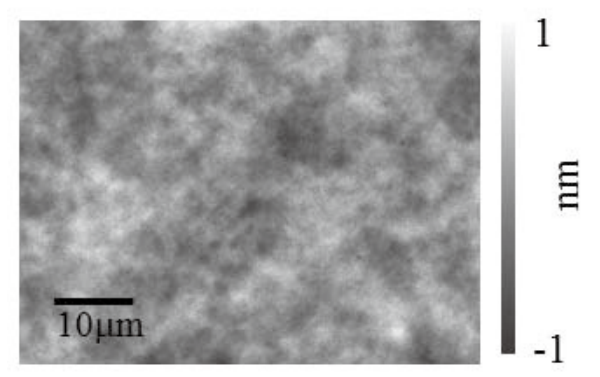

(b) Replicated surface

Fig. 7 Evaluation of repeatability of the electroforming process. (a) Surface height map after the second replication process. The master surface was cleaned by an SPM process. (b) Surface height map of replicated surface obtained by the second process. These maps were obtained by phase-shift microscopic interferometry.

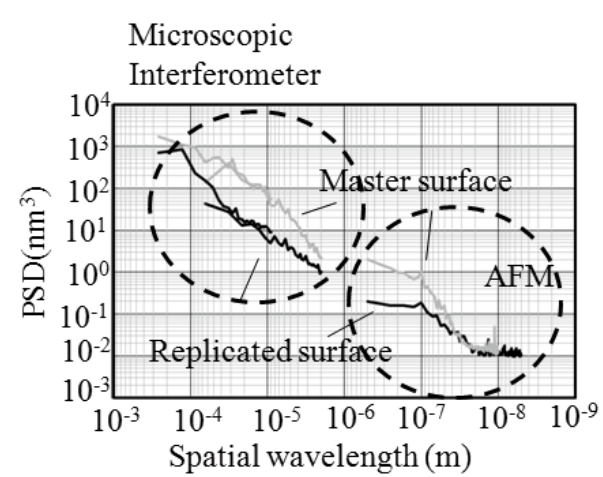

Fig. 8 Power spectral density analysis of the master and replicated surfaces.

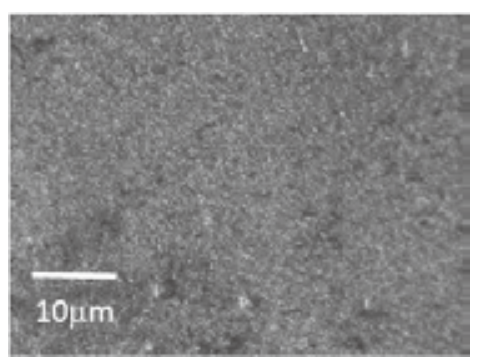

Fig. 9 Optical microscope image of the surface of an electrodeposited film.

an RMS roughness value on the order of $0.2 \mathrm{~nm}$ can be replicated. Figure 8 summarizes all the measured data in figs. 3 through 5 in terms of power spectral density (PSD) analysis. Although, there are no specific peaks in the PSD profiles of the replicated surface, flatness is degraded in the spatial wavelength range from $10^{-7}$ to $10^{-5} \mathrm{~m}$.

In general, the mode of electrodeposition of a metal is known to be island growth. The electrodeposited film is composed of a large number of microcrystals. Figure 9 shows an optical microsocope image of the electrodeposited film, wherein the size of each crystal appears to be a few micrometers. It is known that internal stress remains in the electrodeposited film. The pattern of the internal stress distribution corresponds to the size of the microcrystal. The stress affects the surface morphology of the replicated film. To improve the process, the experimental conditions of electrodeposition need to be adjusted so that the microcrystals are as small as possible.

The flatness of the replicated films corresponds to that of optical surfaces used in many advanced optical devices. This process is also applicable to the replication of nanometer-scale patterned surfaces with $1 \mathrm{~nm}$ height accuracy. We believe that the developed replication process will be widely used in various electroforming processes requiring ultraprecision. 


\section{ACKNOWLEDGMENTS}

This research was mainly supported by PRESTO of the Japanese Science and Technology Agency and partially supported by the Global COE Program, Center of Excellence for Atomically Controlled Fabrication Technology, of the Japanese Ministry of Education, Culture, Sports, Science and Technology.

\section{References}

1) G. Kumar, H. X. Tang and J. Schroers: Nanomoulding with amorphous metals, Nature 457 (12) (2009), pp. 868-872.

2) Hsiharng Yang, Shung-Wen Kang : Improvement of thickness uniformity in nickel electroforming for the LIGA process, International Journal of Machine Tools and Manufacture 40 (2000), pp.1065-1072.

3) L.J. Heyderman, H. Schift, C. David, B. Ketterer, M. Auf der Maur, J. Gobrecht : Nanofabrication using hot embossing lithography and electroforming, Microelectronic Engineering 57-58 (2001), pp. 375-380.
4) Y. Hirai, S. Harada, S. Isaka, M. Kobayashi and Y. Tanaka: Nano-Imprint Lithography Using Replicated Mold by Ni Electroforming, Jpn. J. Appl. Phys. 41 (2002), pp. 4186-4189.

5) J. J. Yang, F. Miao, M. D Pickett, D. A A Ohlberg, D. R Stewart, C. N. Lau and R. Williams: The mechanism of electroforming of metal oxide memristive switches, Nanotechnology 20 (2009), 215201.

6) H. Mimura, H. Ishikura, S. Matsuyama, Y. Sano, K. Yamauchi : Electroforming for replicating nanometer-level smooth surface, Journal of Nanoscience and Nanoterchnology, in press.

7) K. Yamauchi, H. Mimura, K. Inagaki and Y. Mori : Figuring with subnanometer-level accuracy by numerically controlled elastic emission machining, Rev. Sci. Instrum. 73 (2002), pp. 4028-4033.

(Revised February 2011) 